\title{
A convolution approximation property for $L^{\mathrm{I}}(G)$
}

by

L OUIS PIGNO (Manhattan, Kans.)

Abstract. In this paper we define a certain convolution approximation property and give some examples of subspaces which have this property with respect. to $L^{1}(G)$. Wo exhibit a connection between subspaces with the approximation property and multipliors of Fourier transforms. A result on $(p, p)$ multipliers over noncompact LCA groups is also obtained.

Throughout this paper $G$ is a LCA group, $\Gamma$ the group dual to $G$, $M(G)$ the convolution algebra of bounded Borel measures on $G$ and the Fourier-Stieltjes transformation. Denote by $L^{1}(G)$ the space of Haar. integrable functions on $G$ and $D(G)$ any subspace of $L^{1}(G)$ with

$$
D(G) \supset\left\{f \epsilon L^{1}(G): \operatorname{supp} \hat{f} \text { is a compact set }\right\} .
$$

Suppose that whenever $\sigma_{n} \epsilon L^{1}(G)(n=1,2, \ldots)$ with limsup $\left\|\sigma_{n}\right\|_{1}$ $=\infty$ there exists a $k \in D(G)$ such that $\limsup _{n \rightarrow \infty}\left\|\sigma_{n} * k\right\|_{1}=\infty$. Then $D(G)$ is said to have the convolution approximation property with respect to $L^{1}(G)$. It is not difficult to see that $L^{1}(G)$ itself has the convolution approximation property with respect to $L^{1}(G)$; see Example I.

By adapting and generalizing some methods in [1] and [3] we give an example of a rather small space $D(G)$ with the convolution approximation property whenever $G$ is a noncompact LCA group. It turns out that spaces with the convolution approximation property admit a solution to a wide class of multiplior problems. A function $\varphi$ on $\Gamma$ is said to be a multipliter of type $\left(D, L^{1}\right)$ if whenever $f \in D(G)$ there corresponds a. $g \in L^{1}(G)$ such that $p \hat{f}=\hat{g}$.

Triforim 1. If $D(G)$ has the convolution approximation property, then $\varphi \epsilon\left(D, L^{1}\right)$ if and only if $p=\hat{\mu}$ for some $\mu \epsilon M(G)$.

Proof. One half of the Theorem is obvious. For the converse let $p \epsilon\left(D, L^{1}\right)$. For any $k_{\epsilon} D(G)$ with supp $\hat{k}$ a compact subset of $\Gamma$ put

$$
\sigma(x)=\int_{i^{+}} p(\alpha) \hat{k}(\alpha) \alpha(x) d \alpha \quad(x \in G) .
$$


Then $\varphi=\hat{\mu}$ for some $\mu \in M(G)$ if and only if no matter what be $k$ (as defined above) there exists an $M \in \boldsymbol{N}$ (the natural numbers) such that

$$
\|\sigma\|_{1} \leqslant M
$$

whenever $\|k\|_{1} \leqslant 2$. To prove sufficiency let $p(x)=\sum_{i=1}^{n} c_{\alpha_{i}} a_{i}(x)$ be any trigonometric polynomial on $G$ with $\|p\|_{\infty} \leqslant 1$. Now by [4], p. 53, there is a $k \in D(G)$ such that $\hat{k}$ has compact support,

So,

$$
\left\|z_{i}\right\|_{1} \leqslant 2 \quad \text { and } \quad \hat{k}\left(a_{i}\right)=1 \quad(i=1,2, \ldots, n) .
$$

$$
\left|\sum_{i=1}^{n} c_{\alpha_{i}} p\left(\alpha_{i}\right)\right|=\left|\sum_{i=1}^{n} o_{\alpha_{i}} \hat{k}\left(\alpha_{i}\right) p\left(\alpha_{i}\right)\right|
$$

and we obtain

$$
\left|\sum_{i=1}^{n} c_{\alpha_{i}} \varphi\left(a_{i}\right)\right|=\left|\sum_{i=1}^{n} c_{a_{i}} \hat{\sigma}\left(\alpha_{i}\right)\right| .
$$

Thus

$$
\left|\sum_{i=1}^{n} c_{\alpha_{i}} \varphi\left(\alpha_{i}\right)\right| \leqslant\|p\|_{\infty}\|\sigma\|_{1} \leqslant\|\sigma\|_{1} .
$$

iSo

$$
\left|\sum_{i=1}^{n} c_{\alpha_{i}} p\left(\alpha_{i}\right)\right| \leqslant M
$$

for all trigonometric polynomials $p(x)=\sum_{i=1}^{n} c_{\alpha_{i}} \alpha_{i}(x)$ with $\|p\|_{\infty} \leqslant 1$ and this in turn implies by the Bochner-Eberlein Theorem ([4], p. 32) that $\varphi=\hat{\mu}$ for some $\mu \in M(G)$ since $\varphi$ is continuous.

Let us now suppose that $\varphi \notin M(G)^{\wedge}$. We shall force a contradiction: By the preceding argument there exists a sequence $k_{n} \in D(G)$ such that for each $n \in \boldsymbol{N},\left\|k_{n}\right\|_{1} \leqslant 2, \hat{k}_{n}$ has compact support and

$$
\sigma_{n}(x)=\int_{\Gamma} \hat{k}_{n}(\alpha) p(\alpha) \alpha(x) d \alpha \quad(x \in G)
$$

satisfies

$$
\limsup _{n \rightarrow \infty}\left\|\sigma_{n}\right\|_{1}=\infty
$$

Since $D(G)$ has the convolution approximation property, there is a $k \in D(G)$ such that

$$
\limsup _{n \rightarrow \infty}\left\|\sigma_{n} * k_{k}\right\|_{1}=\infty
$$

Observe that

$$
\left(\sigma_{n} * k\right)(x)=\int_{\Gamma} \hat{h}(\alpha) \hat{k}_{n}(\alpha) \alpha(x) d \alpha
$$

for some $h \epsilon L^{1}(G)$ since $\phi \epsilon\left(D, L^{1}\right)$. Thus by the Inversion Theorem $([4]$, p. 22)

$$
\sigma_{n} * \hbar=h * k_{n} \text {. }
$$

Inasmuch as $\left\|h * 7 c_{n}\right\|_{1} \leqslant 2\|h\|_{1}$ we have

$$
\limsup _{n \rightarrow \infty}\left\|\sigma_{n} * k\right\|_{1} \leqslant 2\|h\|_{1} .
$$

Since (ii) says, in particular, that limsup $\left\|\sigma_{n} * k\right\|_{1}<\infty$ this contradicts (2) and completes the proof.

We next present some examples.

ExAmpre I. Let $G$ be a LOA group. Then $L^{1}(G)$ has the convolution approximation property with respect to $L^{1}(G)$. This follows immediately from the Uniform Boundedness Principle and the fact that $L^{1}(G)$ has a bounded approximate identity.

As a consequence of Theorem 1 we obtain that $\left(L^{1}, L^{1}\right)=M(G)^{\wedge}$; this is, of course, well known. Our next example is more interesting.

Exampre II. For $G$ a noncompact LOA group put

$$
A^{0}(G)=\left\{f \in L^{1}(G): \hat{f} \epsilon \bigcap_{0<p<\infty} L^{p}(T)\right\} .
$$

Then $A^{0}(G)$ has the convolution approximation property with respect to $L^{1}(G)$. To see this fix $f \in L^{1}(G)$. Now given $\varepsilon>0$ there is a $k \in L^{1}(G)$ such that: $\hat{k}$ has compact support;

$$
\|k * f-f\|_{1} \leqslant \varepsilon / 2 \quad \text { and } \quad\|k\|_{1} \leqslant 1
$$

For $K * f \in L^{1}(G)$ there is a $g \in L^{1}(G)$ such that $g$ has compact support $K$ and $\|g-k * f\|_{1} \leqslant \varepsilon / 2$.

Then, if we assume that $G$ contains a copy of $Z$, it follows by the noncompactness of $G$ that there is an $a \epsilon G$ such that the sets

$$
n a+K \quad(n \in \boldsymbol{Z})
$$

are pairwise disjoint. Following [3], p. 129, put

$$
k_{n}(x)=\frac{1}{n} \sum_{i=0}^{n-1} k_{i a}(x) \quad(x \in G) .
$$

Then for some $\theta$ and all $n \in \boldsymbol{N}$

$$
\|f\|_{1}=\left\|k_{n} * f\right\|_{1}+\theta \varepsilon \quad(|\theta| \leqslant 1) .
$$


Now

$$
\hat{k}_{n}(\gamma)= \begin{cases}\frac{1}{n}\left(\frac{1-\overline{\gamma(a)^{n}}}{1-\overline{\gamma(a)}}\right) \hat{k}(\gamma), . & \gamma(a) \neq 1, \\ \hat{k}(\gamma), & \gamma(a)=1 .\end{cases}
$$

Since the annihilator of the copy of $\boldsymbol{Z}$ has Haar measure zero in $\Gamma$, the expression above shows $\hat{k}_{n}(\gamma) \rightarrow 0$ a.e. Notice that $\left|\hat{k}_{n}(\gamma)\right| \leqslant|\hat{k}(\gamma)|$, hence it follows by the Lebesgue Dominated Convergence Theorem that

$$
\left\|\hat{k}_{n}\right\|_{p} \rightarrow 0 \quad(0<p<\infty) .
$$

Thus when $G$ contains a copy of $\boldsymbol{Z}$ we have that given $\sigma_{n} \in L^{1}(G)$ and $\varepsilon_{n}>0(n \in \boldsymbol{N})$ there correspond $g_{n} \in A^{0}(G)$ such that

$$
\left\|\sigma_{n}\right\|_{1}=\left\|\sigma_{n} * g_{n}\right\|_{1}+\theta_{n} \varepsilon_{n} \quad\left(\left|\theta_{n}\right| \leqslant 1\right) .
$$

Furthermore, the $g$ 's in (5) may be chosen to satisfy

$$
\left\|\hat{g}_{n}\right\|_{1 / n} \leqslant 1 \text { and }\left\|g_{n}\right\|_{1} \leqslant 1 .
$$

Using structure theory for LCA groups it can be shown that (5) and (6) hold for every noncompact LCA group.

Observe that since $p$ may lie in $(0,1)$, Banach space techniques are not available to us (cf. Theorem 3.1 of [2], p. 188). To show that $A^{0}(G)$ has the convolution approximation property we modify and generalize an argument of Doss ([1], pp. 184-185) given for $G=\boldsymbol{R}$. We reproduce some details from [1] for the readers' convenience.

Suppose that $\sigma_{n} \in L^{1}(G)$ and

$$
\limsup _{n \rightarrow \infty}\left\|\sigma_{n}\right\|_{1}=\infty \text {. }
$$

In (5) set $\varepsilon_{n}=\frac{1}{4}\left\|\sigma_{n}\right\|_{1}$ for all $n \in \boldsymbol{N}$.

Let $\left\langle g_{n}\right\rangle_{1}^{\infty}$ satisfy (5) and (6). For any subsequence $\left\langle g_{n_{q}}\right\rangle_{1}^{\infty}$ define

$$
g(x)=\sum_{q=1}^{\infty} g_{n_{q}}(x) / 3^{q} \quad(x \in G)
$$

Since by (6) $\left\|g_{n_{\alpha}}\right\|_{1} \leqslant 1$ for all $n_{q}$, we conclude that $g \in L^{1}(G)$ and. $\|g\|_{1} \leqslant 1$. We now prove that $\hat{g} \epsilon \bigcap_{0<p<\infty} I^{p}(\Gamma)$.

Given $p \in(0, \infty)$ choose $n \in \boldsymbol{N}$ such that $1 . / n<p$. Then $\left\|\hat{g}_{m}\right\|_{1 / n}^{1 / n} \leqslant 1$ for all $m \geqslant n$ since $\left\|\hat{g}_{m}\right\|_{\infty} \leqslant 1$. Put

$$
\hat{s}_{N}(\gamma)=\sum_{q=1}^{N} \hat{g}_{n_{q}}(\gamma) / 3^{q}
$$

Recall that for $f_{1}$ and $f_{2} \epsilon L^{1 / n}(\Gamma)$

$$
\left\|f_{1}+f_{2}\right\|_{1 / n}^{1 / n} \leqslant\left\|f_{1}\right\|_{1 / n}^{1 / n}+\left\|f_{2}\right\|_{1 / 2}^{1 / n} .
$$

Thus for all $N>n$

$$
\left\|\hat{s}_{N}\right\|_{1 / n}^{1 / n}<\sum_{j=1}^{n}\left(\frac{1}{3}\right)^{j / n}\left\|\hat{g}_{n_{j}}\right\|_{1 / n}^{1 / n}+1 / 3\left(1 / 3^{1 / n}-1\right) .
$$

Inasmuch as $\hat{s}_{N}$ converges uniformly to $\hat{g}$ we have by the Fatou Lemma that $\hat{g} \epsilon L^{1 / n}(\Gamma)$ and this implies since $\|\hat{g}\|_{\infty} \leqslant 1$ that $\hat{g} \in L^{p}(T)$.

We shall show there is a subsequence of $\left\langle g_{n}\right\rangle_{1}^{\infty}$ such that lim sup $\left\|g * \sigma_{n}\right\|_{1}$ $=\infty$ where $g$ is defined by (8): Observe that if, for some $k_{k} \boldsymbol{N} \boldsymbol{N}, g_{k}$ satisfies

$$
\limsup _{n \rightarrow \infty}\left\|g_{k} * \sigma_{n}\right\|_{1}=\infty
$$

then we are done. So suppose that given $k \in \boldsymbol{N}$ there corresponds an $M_{k} \in \boldsymbol{N}$ such that for all $n \in \boldsymbol{N}$

$$
\left\|g_{k} * \sigma_{n}\right\|_{1} \leqslant M_{k}
$$

For any sequence $\left\langle g_{n_{q}}\right\rangle_{1}^{\infty}$ and corresponding $g$ defined by (8) we have

$$
\left\|g * \sigma_{n_{q}}\right\|_{1} \geqslant \frac{1}{3^{q}}\left\|\sigma_{n_{q}} * g_{n_{q}}\right\|_{1}-\sum_{i<q} \frac{1}{3^{i}}\left\|\sigma_{n_{q}} * g_{n_{i}}\right\|_{1}-\sum_{i>q} \frac{1}{3^{i}}\left\|\sigma_{n_{Q}} * g_{n_{i}}\right\|_{1} .
$$

From (5) and our choice of $\varepsilon_{n}$

$$
\left\|g * \sigma_{n_{q}}\right\|_{1} \geqslant \frac{3}{4} \frac{\left\|\sigma_{n_{q}}\right\|_{1}}{3^{q}}-\sum_{i<q} \frac{\left\|\sigma_{n_{q}} * g_{n_{i}}\right\|_{1}}{3^{i}}-\sum_{i>q} \frac{1}{3^{i}}\left\|\sigma_{n_{q}} * g_{n_{i}}\right\|_{1}
$$

and by (9)

$$
\left\|g * \sigma_{n_{q}}\right\|_{1} \geqslant \frac{3}{4} \frac{\left\|\sigma_{n_{q}}\right\|_{1}}{3^{q}}-\sum_{i<q} \frac{M_{n_{i}}}{3^{i}}-\sum_{i>q} \frac{1}{3^{i}}\left\|\sigma_{n_{q}} * g_{n_{i}}\right\|_{1} .
$$

Finally, since

$$
\left\|\sigma_{n_{q}} * g_{n_{i}}\right\|_{1} \leqslant\left\|g_{n_{i}}\right\|_{1}\left\|\sigma_{n_{q}}\right\|_{1}
$$

we have via (6) the inequality

Thus,

$$
\left\|g * \sigma_{n_{\alpha}}\right\|_{\mathbf{1}} \geqslant \frac{3}{4} \frac{1}{3^{\alpha}}\left\|\sigma_{n_{Q}}\right\|_{1}-\sum_{i<q} \frac{M_{n_{i}}}{3^{i}}-\left\|\sigma_{n_{q}}\right\|_{1} \sum_{i>Q} \frac{1}{3^{i}}
$$

$$
\left\|g * \sigma_{n_{q}}\right\|_{1} \geqslant \frac{1}{4} \frac{\left\|\sigma_{n_{q}}\right\|_{1}}{3^{q}}-\sum_{i<q} \frac{M_{n_{i}}}{3^{i}} ;
$$

and this inequality holds for all subsequences $\left\langle g_{n_{q}}\right\rangle_{1}^{\infty}$. Inasmuch as $\underset{n \rightarrow \infty}{\limsup }\left\|\sigma_{n}\right\|_{1}=\infty$, (11) permits the construction of a subsequence $\left\langle g_{n_{q}}\right\rangle_{1}^{\infty}$ such that $g$ satisfies $\limsup _{n \rightarrow \infty}\left\|g * \sigma_{n}\right\|_{1}=\infty$. Our discussion is complete. 
As a consequence of our Theorem it follows that when $G$ is a noncompact LCA group $\varphi \in\left(A^{0}, L^{1}\right)$ if and only if $\varphi=\hat{\mu}$ for some $\mu \epsilon M(G)$, cf. Theorem 3.1 of [2], p. 188. This result has also been obtained by S. Saeki (private communication). Notice that $A^{0}(G)$ does not have the convolution approximation property when $G$ is compact since $\left(L^{2}, L^{2}\right)=L^{\infty}(I)$.

For $G$ a noncompact LOA group and $1 \leqslant p \leqslant 2$ put

$$
A_{1}^{p}(G)=\left\{f \in L^{p}(G): \hat{f} \in L^{1}(T)\right\} .
$$

Recall that $A_{1}^{p}(G)$ is a Banach space when equipped with the norm $\|f\|_{p}+$ $+\|\hat{f}\|_{1}, f \in A_{1}^{p}(G)$. Denote by $\left(A_{1}^{p}, L^{p}\right)$ the Banach space of all bounded linear maps from $A_{1}^{p}(G)$ to $I^{p}(G)$ which commute with translation.

By $M_{p}^{p}(G)$ we mean the space of all continuous linear operators from $L^{p}(G)$ to $L^{p}(G)$ which commute with translation. The proof of the following result may be of some interest and does not seem to be widely known:

THeonem 2. For $G=\boldsymbol{R}$ the operator $T \epsilon\left(A_{1}^{p}, L^{p}\right)$ if and only if $T$ extends to an operator in $M_{p}^{p}(\boldsymbol{R})$.

Proof. One half of the Theorem is obvious. For the converse we have by the continuity of $T$ a constant $K>0$ such that

$$
\|T(f)\|_{p} \leqslant K\left\{\|f\|_{p}+\|\hat{f}\|_{1}\right\} \quad\left(f \in A_{1}^{p}(\boldsymbol{R})\right) .
$$

Let $f \epsilon A_{1}^{p}(\boldsymbol{R})$, then

$$
\lim _{a \rightarrow \infty}\left\|f+\frac{1}{2} f_{a}+\frac{1}{2} f_{-a}\right\|_{p}=\left(1+1 / 2^{p-1}\right)^{1 / p}\|f\|_{p}
$$

and since $T$ is linear and commutes with translation

$$
\lim _{a \rightarrow \infty}\left\|T\left(f+\frac{1}{2} f_{a}+\frac{1}{2} f_{-a}\right)\right\|_{p}=\left(1+1 / 2^{p-1}\right)^{1 / p}\|T(f)\|_{p} .
$$

Now

$$
\left(f+\frac{1}{2} f_{a}+\frac{1}{2} f_{-a}\right)^{\wedge}(\alpha)=\left(1+\frac{\alpha(a)}{2}+\frac{\overline{\alpha(a)}}{2}\right) \hat{f}(\alpha)
$$

and since the kernel $\left(1+\frac{a(a)}{2}+\frac{\overline{a(a)}}{2}\right)$ is non-negative, we obtain by the Riemann-Lebesgue Lemma that

$$
\lim _{a \rightarrow \infty} \int_{\boldsymbol{R}}|\hat{f}(\alpha)|\left|1+\frac{\alpha(a)}{2}+\frac{\overline{\alpha(a)}}{2}\right| d \alpha=\int_{\boldsymbol{\varkappa}}|\hat{f}(\alpha)| d \alpha .
$$

We conclude therefore that

$$
\|T(f)\|_{p} \leqslant K\left\{\|f\|_{p}+\left(1+1 / 2^{p-1}\right)^{-1 / p}\|\hat{f}\|_{1}\right\} .
$$

Iterating the process $n$ times we have

$$
\|T(f)\|_{p} \leqslant K\left\{\|f\|_{p}+\left(1+1 / 2^{p-1}\right)^{-n / p}\|\hat{f}\|_{1}\right\} .
$$

Well, this means since $n \in \boldsymbol{N}$ is arbitrary that

$$
\|T(f)\|_{p} \leqslant K\left\{\|f\|_{p}\right\} \quad\left(f \in A_{1}^{p}(\boldsymbol{R}) .\right.
$$

Since $A_{1}^{p}(\boldsymbol{R})$ is dense in $L^{p}(\boldsymbol{R})$ we may extend $T$ in the desired fashion. This completes the proof.

It is easy to see that the proof of Theorem 2 generalizes to a wide class of noncompact LOA groups.

\section{References}

[1] R. Doss, On the multiplioators of some classes of Fourier transforms, Proc. London Math. Soc. (2) 50 (1949), pp. 169-195.

[2] A. Figà-Talam anca and G. I. Gaudry, Multiplier and sets of uniqueness of $L^{p}$, Michigan Math. J. 17 (1970), pp. 179-191.

[3] G. I. Gaudry, Topics in Harmonic Analysis, Lecture Notes Dept. of Mathematics, Yale University, 1969.

[4] W. Rudin, Fourier Analysis on Groups, Intersoience, New York 1962.

KANSAS STATEE UNTVRRSITY 